\title{
EchoGéo
}

31 | 2015

Glocal Ethiopia

\section{Armanya : l'oignon, le masho, la bonne, le « koulak » et le prolétaire}

\section{René Lefort}

\section{OpenEdition}

\section{Journals}

Édition électronique

URL : https://journals.openedition.org/echogeo/14161

DOI : 10.4000/echogeo.14161

ISSN : 1963-1197

\section{Éditeur}

Pôle de recherche pour l'organisation et la diffusion de l'information géographique (CNRS UMR 8586)

\section{Référence électronique}

René Lefort, «Armanya : l'oignon, le masho, la bonne, le « koulak » et le prolétaire », EchoGéo [En ligne], 31 | 2015, mis en ligne le 10 avril 2015, consulté le 31 juillet 2021. URL : http://

journals.openedition.org/echogeo/14161; DOI : https://doi.org/10.4000/echogeo.14161

Ce document a été généré automatiquement le 31 juillet 2021.

EchoGéo est mis à disposition selon les termes de la licence Creative Commons Attribution - Pas d'Utilisation Commerciale - Pas de Modification 4.0 International (CC BY-NC-ND) 


\title{
Armanya : l'oignon, le masho, la bonne, le « koulak » et le prolétaire
}

\author{
René Lefort
}

\section{Du « poor farmer » au « model farmer »}

1 À première vue, c'est un village - désormais un bourg avec ses 2000 habitants environ comme tous ceux qui peuplent le haut plateau amhara ${ }^{1}$. À quelque deux cents kilomètres au nord-est d'Addis-Abeba, le kebele ${ }^{2}$ dont il est le chef-lieu s'étage au long de la cassure qui mène des hautes terres du plateau abyssin à la dépression afar. Plutôt moins pauvre que la moyenne: il n'est pas, et il n'était pas, éligible à l'un des programmes d'assistance, dont le fameux Productive Safety Net Programme ${ }^{3}$. Il abrite une école primaire récemment agrandie, un impressionnant centre de santé tout neuf et une nouvelle gendarmerie: le «Developmental state» éthiopien prouve une fois de plus son efficacité à construire des infrastructures, notamment pour l'éducation et la santét ${ }^{4}$ La coopérative, dont la principale fonction est d'approvisionner les paysans en engrais, est toute proche du bureau des trois inévitables " agents de développement " (culture, bétail, environnement). Mais Armanya ${ }^{5}$ a une particularité : une grande route le traverse, celle qui va d'Addis-Abeba aux régions nord-est du Wollo puis du Tigray. L'ouverture qu'elle offre sur l'Éthiopie et, au-delà, sur le monde, a révolutionné l'économie, la société, la structure politique, à plus forte raison après que le régime a décidé de changer radicalement de cap, au tout début des années $2000^{6}$.

Voilà seulement une douzaine d'années, Armanya continuait sur la lancée de la réforme agraire de 1975, mise en place par le régime précédent - la junte « socialiste » du Derg -. Dès ses débuts, elle avait matérialisé le mot d'ordre « land to the tiller » et donc décapité la noblesse terrienne et annulé l'emprise foncière de l'Église. En 1997, six ans après leur prise de pouvoir ${ }^{7}$, les nouvelles autorités décidaient une autre redistribution des terres en région amhara, dans un double but, économique et politique. Ceux qui étaient trop jeunes pour avoir bénéficié de la précédente, désormais adultes, recevaient ainsi leur juste part de terre. En contrepartie, le pouvoir misait sur eux pour bâtir une nouvelle 
assise rurale. À l'opposé, l'élite traditionnelle était subrepticement dépossédée, ouvertement vilipendée et constamment ostracisée au motif qu'elle avait monopolisé les fonctions officielles au temps du Derg ${ }^{8}$. D'où son surnom de birokrasi.

Quoi qu'il en soit, l'homogénéité et l'isolement étaient les traits majeurs de ce monde rural. Peu ou prou, tous cultivaient des superficies du même ordre de grandeur - un hectare environ'-, les mêmes produits essentiellement auto-consommés, avec les mêmes méthodes, mis à part une poignée de commerçant en grains, également prêteurs, et une douzaine de fonctionnaires, dont les instituteurs. On vendait au marché hebdomadaire quelques surplus locaux pour acheter du sel, du pétrole pour la lampe, des allumettes, un short ou une chemise. La seule télévision trônait dans la gargote la plus fréquentée. Le mot d'ordre national était l'autosuffisance alimentaire de chaque foyer, la cible le "poor farmer ", que l'accroissement de sa productivité devait conduire dans un pays de Cocagne. "In the villages, the houses will be chic and modern. Each dwelling will have electric power, telephone and running water... modern beds, chairs, tables and other household utensils. They will all have radio, television and modern electronic instruments $\|^{10}$.

4 Peine perdue : la productivité stagnait, les urgences alimentaires se perpétuaient. En même temps, le pays affrontait successivement deux profondes crises politiques. En 2001, une partie de la direction historique était expulsée du TPLF, suivie par une cohorte de responsables et militants, accusées de "gauchisme». En 2005, le régime prenait une retentissante claque électorale, totalement inattendue, pour laquelle la birokrasi avait joué un rôle décisif en région amhara. Il ne s'en sortait que par la fraude et la répression. La combinaison de ces deux chocs allait conduire à un virage économique à 180 degrés complètement imbriqué dans une refondation politique. La survie de l'Éthiopie ne pourrait passer que par l'économie de marché et l'insertion dans la compétition mondiale. Pour l'agriculture en particulier - un peu moins de la moitié du PIB et les 4/5èmes de la population active -, la stratégie "pro-poor », qui revenait à « détacher les paysans de la commercialisation » aurait été erronée. Il fallait désormais «se concentrer sur l'économie de marché en tant que centre des activités rurales ». Le moteur de la croissance agricole ne serait plus le "semi-subsistance small farmer" mais "l'initiative privée " de celui capable de "to intensify marketable farm products». Ce segment supérieur, promu "fermier modèle ", était enrôlé, de gré ou de force, dans le parti quasi unique pour devenir la nouvelle base politique du régime dans les campagnes. En échange, il recevrait toute l'attention et tout le soutien de la puissance publique. La masse des agriculteurs était, de fait, plus ou moins abandonnée à son sort, en clair aux lois du marché ${ }^{11}$.

\section{La « révolution de l'oignon »}

Dire que les «model farmers " ne voyaient aucun intérêt à cette promotion serait un euphémisme. Ils étaient viscéralement opposés à un régime qui les avait spoliés en 1997 et passait son temps à les clouer au pilori. Ils jugeaient que la structure mise en place pour le développement agricole n'était qu'une lourde entrave, tout comme leur appartenance forcée au quasi parti unique, avec comme corollaires de l'une et de l'autre des réunions aussi inutiles qu'interminables et fréquentes. Bref, ils adhéraient au credo du paysan éthiopien depuis des lustres : plus on se tient à distance du pouvoir, plus on échappe à son oppression et à sa prédation. 
6 Ce n'est d'ailleurs pas par son intermédiaire qu'Armanya a connu sa première révolution agricole depuis l'introduction des engrais. À quelques dizaines de kilomètres en contrebas, au bord de la rivière, existait une forte tradition de maraîchage, et en particulier de la culture intensive de l'oignon. Un premier paysan a essayé, ses voisins l'ont observé, ils se sont lancés à leur tour, en bref le bouche à oreille et l'observation directe ont conduit cette culture à se répandre comme une trainée de poudre. Mais dans une enquête effectuée alors, tous les paysans qui s'y adonnaient affirmaient ne pas avoir reçu la moindre aide ou conseil des agents de développement en ce domaine ${ }^{12}$.

7 Cette première culture de rente était hautement profitable, même si sa multiplication en a fait baisser les revenus. Compte tenu de la forte intensité de main d'œuvre nécessaire, un foyer en cultive en général entre $1 / 16^{\text {ème }}$ et $1 / 8^{\text {ème }} d$ 'hectare. À la vente, le rapport pour cette surface se situe entre deux cents et quatre cents dollars. Soit, à superficie égale, quatre fois plus que pour la céréale la plus chère.

8 Les effets de ce boom sautent aux yeux. Au temps de la récolte, tous les jours en fin d'après-midi, des groupes de dromadaires affluent devant les marchands - ils ne sont désormais pas loin d'une trentaine - avec leur charge de 200 à 250 kilos contre 50 kilos au maximum pour un âne. On n'en voyait jamais auparavant. Les paysans des basses terres ont saisi l'opportunité de s'employer comme transporteurs grâce à leurs animaux de bât. Une dizaine, parfois une vingtaine de camions attendent leur chargement. La nuit tombée, ils partiront jusqu'à Addis-Abeba ou tout au Nord, Mekelle, la capitale du Tigray.

\section{Le masho, « l'or vert »}

9 La deuxième révolution dans les cultures de rente est toute récente : elle date de quatre ans. Le président du kebele, aussi énergique que redouté, a appris l'existence du masho, le haricot mungo, et comment le cultiver, lors d'un stage de formation. Ils sont réservés en général aux agriculteurs les plus performants et organisés par les services publics de développement agricole. Cette plante, qui ressemble à une petite lentille vert clair, n'était ni connue, ni cultivée, ni consommée en Éthiopie. Elle avait fait son apparition dans le Wollega, le Wollo, le Tigray. Son principal marché se situe en Asie occidentale et, un peu, dans la Péninsule arabe. En faisant visiter son champ expérimental, le président était aux anges, criant à qui voulait l'entendre : «c'est de l'or vert »!

10 La suite lui a donné raison. Le masho présente tous les avantages. Il se cultive à la petite saison des pluies, à cheval sur la fin de l'hiver et le début printemps, quand auparavant les paysans ne tiraient de cette récolte qu'environ le dixième de leur production annuelle. Il n'exige qu'un seul passage d'araire, contre jusqu'à six ou sept pour la céréale locale la plus appréciée, le teff. Il n'est pas gourmand en engrais, apporte du fourrage et nourrit le sol en azote. Avec un rendement de 4 à 6 quintaux à l'hectare et un prix de vente, au printemps 2014, autour de 125 dollars le quintal, la petite récolte peut ainsi rapporter environ 600 dollars par an et par hectare. À supposer qu'un paysan sème pour la grande récolte la même surface uniquement de teff, la céréale la plus rentable - ce qu'il ne fera jamais, pour minimiser les risques -, la valeur de celle-ci serait d'environ 700 dollars. En clair, le masho permet un quasi doublement de la valeur de la production paysanne. 

champs en étaient couverts. Les paysans aiment à dire : « quand on a vendu son masho, on est tellement content qu'on a envie d'offrir une bière à son âne!».

\section{Les « Américains »}

Ils sont les enfants de ces deux révolutions. Ils paradent, casquette à l'envers, tee-shirt flashy, lunettes de soleil genre Ray Ban. Ils fument, ce qu'aucun paysan ne fait. Ils sont les piliers des gargotes du village, où ils boivent et mangent à satiété presque quotidiennement, ce que même un paysan « riche $»^{13}$ ne se permet pas.

13 Ils étaient, au départ, totalement désœuvrés. Enfants de fermiers pauvres, sans terre, ou de femmes chefs de foyer, originaires en général des confins du kebele et même de kebele assez éloignés, peu ou pas du tout éduqués, ils faisaient partie de ces masses de jeunes qui, comme on le dit en Algérie, passaient leur journée à « tenir les murs » (voir plus loin la partie sur les prolétaires).

14 La formation de ce groupe est venue de l'initiative du chef de la police du village. Comme toutes les autorités locales, sa hantise est - et reste - que des jeunes, chômeurs et/ou sans terre ne créent des «troubles». L'embauche pour le chargement de chaque camion dégénérait en bagarres. Le chef décida donc d'y mettre de l'ordre. La sélection s'est faite informellement, parmi ceux qui étaient les plus débrouillards et savaient le mieux jouer des coudes. Ils sont désormais 43 , pas un de plus, pas un de moins, à en avoir le monopole. Ils ont nécessairement dû devenir membres du Parti même si, pour dire le moins, ils ne manifestent pas un militantisme acharné. Ils se sont cooptés, et s'auto organisent pour se répartir le travail, payés à la tâche : 0,15 dollars par sac transporté. En complément, ils vont de temps à autre donner un coup de main dans l'exploitation familiale - quand elle existe -, ou s'emploient comme journalier. Si les villageois ne les situent pas bien haut dans l'échelle sociale, les « Américains »- leur surnom - tiennent, au sens littéral, le haut du pavé. Leurs revenus sont certes irréguliers mais bien peu les égalent, hormis les marchands et les paysans les plus « riches ». Ils forment une sorte d'aristocratie des travailleurs occasionnels ; ils sont les rois du « petit boulot ».

\section{Les « housemaids »}

Les «Américains » ont leur pendant féminin. Elles aussi remontent et descendent la grande route, mais bras dessus bras dessous, regardant à la dérobée si elles font bien leur effet. Elles sont devenues plus qu'à l'aise, et le montrent : des vêtements neufs et chatoyants, loin des robes longues et ternes de la plupart des femmes, des bijoux en or - ou qui y ressemblent -, un maquillage prononcé, des coiffures apprêtées...

Ce mouvement de migration féminine temporaire a commencé voilà une dizaine d'années, essentiellement vers les pays du Golfe, un peu vers le Liban. Presque toujours légal, il touche essentiellement la tranche d'âge des 20-35 ans. Les contrats sont habituellement de deux ans, après lesquels ces femmes retournent quelques semaines au bourg puis repartent. L'élasticité de la structure familiale permet qu'elles n'aient aucune difficulté à confier leurs enfants à des parents, proches ou lointains. Les salaires mensuels, autour de 200 dollars, sont mirobolants à l'aulne locale : seul un paysan très

EchoGéo, 31 | 2015 
« riche » peut prétendre à un revenu équivalent. Elles n'ont donc aucune peine à faire des émules.

17 Combien d'entre elles sont hors du bourg à un moment donné? Les autorités locales parlent de 50 à 100, sur une population totale qu'elles estiment à $6000^{14}$. Et lorsqu'on interroge une douzaine de ces femmes sur la dureté de leur emploi - en général douze heures par jour, six jours par semaine, le plus souvent sans pouvoir sortir de la maison pendant des mois -, toutes répondent avec une teinte de stupéfaction : "parce que vous ne voyez pas que c'est pire ici? Et en plus on ne gagne rien! ». Volonté de faire bonne figure? Ou réponse sincère, tant les multiples tâches des femmes sont exténuantes?

\section{Les « koulaks »}

La «birokrasi» a pris sa revanche. Plus éduquée, souvent plus innovatrice et entreprenante, elle a joué à plein de son capital social, à peine entamé, et de la survalorisation des «fermiers modèles » par les autorités, pour tirer le meilleur parti de ces révolutions agricoles.

Forte de sa réputation de «strong farmer», c'est vers elle que se sont tournés en priorité les personnes âgées, les veuves, les malades, les femmes dont le mari était parti tenter sa chance en ville, pour tirer le profit maximal d'un lopin de terre qu'ils ne pouvaient cultiver. Ils l'ont loué ou mis en métayage. Ces "strong farmers " ont ainsi obtenu un premier agrandissement de leurs exploitations et, du même coup, quelques économies en liquide. Parallèlement, la décision des autorités de ne plus procéder à une redistribution des terres, la croissance démographique, les divisions par héritage, conjuguées à l'absence totale de nouvelles terres à défricher, ont multiplié le nombre des paysans potentiels mais sans terre ou de paysans possédant une parcelle beaucoup trop petite pour en vivre : ceux qui avaient moins de dix-huit ans en 1997 ont été exclus de la dernière redistribution agraire; ils sont désormais adultes. Soit ils ont mis ces parcelles en métayage ou en location, soit ils ont tenté de les exploiter mais, souvent, pour finir par sombrer dans la spirale de l'endettement. Pris à la gorge, ils ne pouvaient se tourner que vers ces " cumulards", les seuls dotés des moyens de payer comptant la location de leur lopin ou de leur permettre de rembourser leur dette. De 1997 à aujourd'hui, certains de ces nouveaux « koulaks » ont ainsi multiplié par quatre à cinq la superficie de leur exploitation.

À première vue, rien ne les distingue d'un paysan ordinaire. Sauf le téléphone portable, le symbole de leur réussite, qu'ils exhibent en le tenant à la main même s'ils ne peuvent guère l'utiliser à cause de la faible couverture du réseau. Ils ne partagent ce signe ostentatoire qu'avec les fonctionnaires et les marchands. Mais fortune faite grâce à l'agriculture - à l'échelle du bourg -, ils s'en détournent pour investir dans un secteur beaucoup plus rentable, moins risqué et infiniment moins pénible : le tertiaire. Leurs investissements ne vont plus à l'extension de leur exploitation, mais au petit commerce, l'ouverture d'un moulin, l'achat d'un minibus de passagers, et surtout l'immobilier. Ils agrandissent leur maison de deux ou trois chambres qu'ils louent soit à des fonctionnaires, soit, surtout, à des jeunes. Ceux-ci s'y entassent à trois ou quatre, venus tenter leur chance au bourg et/ou fuyant le foyer familial, survivant d'un « petit boulot $»^{15}$.

21 Cet enrichissement conduit à la formation de ce qui s'apparente à une classe moyenne supérieure rurale, bien plus nombreuse que son équivalent urbain et pourtant 
largement ignorée. Son succès économique réaffirme son ascendant social, d'autant que les autorités locales ne cessent de monter celui-là en épingle auprès de la masse de ceux qui restent très loin en arrière. Elle a aussi repris pied dans le pouvoir politique local formellement du moins - puisqu'elle monopolise les postes subalternes de direction du parti, jusqu'au plus bas niveau de l'encadrement de la population, l'unité de cinq foyers, et ceux du conseil municipal, même s'il ne se réunit jamais. Les autorités sont en passe de réussir leur pari. La connivence entre ces nouveaux koulaks et les autorités locales est de plus en plus étroite. Entre autres, elle pèsera décisivement lors des élections à venir, car ils sont plus que jamais les « faiseurs d'opinion ».

\section{Les prolétaires}

En période de grands travaux agricole, au lever de jour, une centaine de femmes et d'hommes sont rassemblées au bord de la grande route, dans l'espoir d'une embauche pour la journée. "On nous choisit comme des bêtes", disent-ils. Cette massification du travail précaire était inimaginable voilà quelques années. Le travailleur journalier existait, bien sûr, mais s'employait via des liens de parenté ou de voisinage, soit dans le cas le plus fréquent d'une entraide réciproque, soit de bouche-à-oreille, entre un fermier « riche» et un paysan voisin «pauvre » ou sans terre. Ceux qui s'y adonnent sont rarement jeunes - n'importe quoi plutôt qu'un travail agricole, le plus déconsidéré -, et souvent des femmes, en majorité des mères célibataires ou des veuves. Cependant, dans les périodes où beaucoup de bras sont requis pour travailler en urgence, les salaires peuvent être très élevés à l'échelle du pays, jusqu'à 5 dollars par jour lors de la moisson d'automne de 2013. Les femmes perçoivent moins.

L'auteur a tenté de mesurer ce phénomène en cherchant à évaluer en 2013 la proportion de "chômeurs » parmi les jeunes hommes (de 16 à 30 ans). Il est parti de leur propre définition de cette situation : celui qui ne peut pas vivre de façon autonome et à plus forte raison fonder un foyer parce qu'il n'a pas de travail, ou un travail trop précaire, ou qu'il n'a pas de terre, ou trop peu. Par trois voies différentes, l'auteur est arrivé au même ordre de grandeur: les trois quarts de cette tranche d'âge sont " chômeurs ». Mieux : l'officiel responsable des jeunes au niveau du woreda a confirmé cette proportion, mesurée par sa propre enquête.

Les autorités locales sont littéralement terrorisées parce qu'elles n'ont pas de prise sur eux : c'est le seul groupe social qui échappe à leur appareil de contrôle. En nombre croissant, souvent frondeurs, de plus en plus fréquemment en rupture familiale, ils forment une sorte de lumpen prolétariat rural dont les autorités redoutent qu'il ne soit une bombe à retardement.

Les efforts pour les faire rentrer dans le rang sont aussi infructueux que, parfois, dérisoires. Elles ont été jusqu'à leur proposer de créer un «donkey parking » où, au prix de 0,25 dollars, les paysans venant au marché du dimanche seraient obligés de " parquer " leurs ânes. Les fermiers ont hurlé de fureur et de rire. L'action principale des autorités en direction de ces « chômeurs » n'aboutit pas : un petit prêt pour monter une "petite affaire ", comme une boutique ou un atelier de réparation de mobiles. L'argent public est trop parcimonieux, les jeunes insuffisamment formés. En général, ces micro affaires périclitent puis disparaissent. boisson et un petit quelque chose à manger quand il participe à l'une de ses rares 
réunions. Sa seule réalisation est la plantation collective d'eucalyptus sur un seizième d'hectare, qui ne poussent même pas tant la terre est mauvaise. On leur met systématiquement en main un donnant-donnant : organisez-vous - sous-entendu dans les structures d'encadrement de la population - et les autorités vous aideront. Ces jeunes ne veulent pas en entendre parler. On pourrait reprendre ce qu'ils disaient déjà en 2005 pour justifier leur refus de s'inscrire sur les listes électorales : «l'État ne fait rien pour nous, nous ne ferons rien pour l'État ». Il est entendu ici comme le pouvoir au sens large, c'est-à-dire toutes les institutions comme toutes les personnes qui le détiennent, ce qu'on appelle le « mengist».

On peut dresser un parallèle intéressant entre cette situation et les observations de Marco di Nunzio sur les "street youths» à Addis Abeba ${ }^{16}$. À Armanya aussi le pouvoir craint intensément les "troubles» que ces jeunes pourraient provoquer, depuis les délits ordinaires qui sont source d'insécurité pour les habitants, qui en rendraient les autorités responsables, jusqu'à une jonction avec l'opposition lorsqu'elle descend dans la rue, comme ce fut le cas pendant la Terreur rouge et après les élections de 2005. Là aussi, le pouvoir corrèle chômage et trouble, et cherche donc une issue à un problème pour lui essentiellement politique en poussant à la création d'emplois. Là aussi, on retrouve un processus identique à celui observé à Armanya pour les "Américains ", quand les autorités d'Addis Abeba ont intégré des jeunes travaillant de façon jusque-là informelle dans des coopératives reprenant leurs fonctions antérieures, comme gardien de parking et "minibus touts ", en échange d'un salaire mensuel. Il est d'ailleurs cocasse de rapprocher la structuration des "parking guys" d'Addis Abeba et la tentative de créer le « donkey parking " à Armanya. En bref, on observe dans les deux lieux le même processus de "formalisation and politization of street life» mené par les autorités, en vue de " further expand the reservoir of individuals directly and indirectly linked to the party ». On relève cependant trois différences essentielles. À Armanya, ce processus ne touche pas la majorité des jeunes désœuvrés, celle qui vit dans la campagne même. La menace qu'ils représenteraient est jugée mineure, tout simplement parce qu'ils ne sont pas structurés en bandes comme dans le bourg ou à Addis Ababa : ils sont atomisés, le plus souvent dispersés dans les foyers respectifs de leurs parents, donc considérés comme relativement inoffensifs, donc relégués hors du champ de préoccupations majeures des autorités. Ensuite, ce processus ne touche même pas la majorité des jeunes "chômeurs" du bourg. Peu d'activités économiques s'y prêtent, et les autorités manquent cruellement de moyens pour en créer. Les jeunes peuvent plus facilement résister à la répression des autorités. Par exemple, le Vagrancy Proclamation Act (2004) qui fait tomber sous le coup de la loi le vagabondage, défini de façon aussi large que flou, et qui potentiellement frapperait la plupart des jeunes chômeurs d'Armanya, n'y est d'aucun effet. Enfin, Marco di Nunzio estime aussi que ce processus permet de «to increase the number of people who were dependent on local government bureaucracy for their survival and thus were expected to support the ruling party when needed ». Il est douteux que cette causalité soit aussi marquée à Armanya. Le rapport de force y est plus équilibré. Chacun y trouve son compte : les jeunes, du travail ; les autorités, un certain calme. Il n'est pas sûr qu'elles puissent leur demander plus, soit un appui effectif au Parti.

Les jeunes d'Armanya adoptent trois attitudes face à ce "chômage", étroitement corrélées à leur position géographique par rapport au bourg, qui à son tour détermine largement leur niveau éducatif. Ceux qui y vivent déjà, parce qu'ils y sont nés ou sont venus s'y installer après avoir quitté le foyer familial, envisagent, comme ils le disent, de créer un «small business» dans une ville voisine plus grande. Le deuxième cercle, 
hors du bourg mais pas trop loin, estime que ses chances de réussir en ville sont bloquées par une éducation insuffisante. Il envisage lui aussi de créer une "petite affaire ", mais sur place. Dans les deux cas, ils butent sur le manque de capital, qu'ils estiment au minimum à un petit millier de dollars, et, ce qu'ils négligent trop souvent, un manque de formation professionnelle. Le troisième cercle enfin vit aux confins $d u$ kebele, et n'a en général jamais fréquenté l'école. Lui n'envisage d'autre échappatoire que l'émigration clandestine vers les pays du Golfe car il estime que la concurrence des jeunes plus instruits lui ferme la porte de tout emploi urbain en Éthiopie.

Les passeurs demandent environ 500 dollars. Famille et amis s'emploient à les réunir, souvent en vendant une partie de leur bétail. Depuis peu, quelques passeurs acceptent d'avancer la somme. Même si les autorités locales affirment tout faire pour dissuader les futurs partants, le système est si rôdé qu'à tout le moins elles ferment les yeux. Certains arrivent à destination et trouvent du travail, certains échouent et reviennent, certains n'arrivent jamais parce qu'ils décèdent en route. Tous ceux qui ont tenté sans réussir affirment avoir vu des compagnons de voyage mourir d'épuisement. Mais ils n'ont qu'un objectif : repartir. «Nous n'avons pas le choix », répètent-ils.

Pourtant, réussir cette émigration clandestine est de plus en plus problématique. À la fin de 2013, les autorités d'Arabie Saoudite ont brutalement expulsé les immigrants clandestins. Addis Abeba en attendait une vingtaine de milliers. Il a fallu mettre en place un pont aérien pour en rapatrier entre 150000 et 200000 . Un budget de 2,6 millions de dollars était prévu pour leur réintégration (soit au mieux 15 dollars par rapatrié). À Armanya, ceux-ci affirment avoir reçu à leur arrivée à Addis-Abeba un billet de bus pour rentrer chez eux, un petit pécule, et surtout une promesse d'aide lors d'une réunion au chef-lieu du woreda. Ils disent toujours attendre qu'elle se concrétise...

31 Les négociations restent en cours entre les pays concernés pour la reprise de cette émigration dans un cadre légal et mutuellement agréé. Mais la misère n'attend pas. L'immigration illégale, au moins celle des hommes, a repris à Armanya, toutefois à une échelle moindre qu'auparavant.

\section{Conclusion}

Armanya constitue-t-il une exception, ou son cas est-il généralisable? Oui et non. L'Éthiopie est trop diverse, les ensembles écologiques trop nombreux pour que l'idée d'un «village moyen " puisse faire sens. Mais la plupart des tendances lourdes qui ont bouleversé ce kebele pendant la dernière décennie sont perceptibles ailleurs, notamment pour ceux d'entre eux qui, sur le plateau abyssin, le kebessa, sont au bord d'une voie de communication ou pas trop loin. Or ils sont de plus en plus nombreux avec la croissance fulgurante du réseau routier ${ }^{17}$.

Entre ces sous-prolétaires ruraux et les paysans "riches ", à plus forte raison les marchands dont les affaires sont florissantes, un monde s'est creusé en un temps incroyablement bref: une douzaine d'années. Pendant ce court délai, Armanya a basculé d'une relative homogénéité à une hétérogénéité flagrante. La foule des travailleurs journaliers se réunit devant des maisons désormais en parpaings, au sol en ciment, meublées de tables et de fauteuils, et surmontées d'antennes paraboliques. Les araki bet, ces bistrots où l'on boit un alcool local, l'araki, ont essaimé. Le dimanche en fin d'après-midi, après le marché, des files de paysans ivres, cramponnés à leur bâton pour ne pas tomber, s'étirent sur les chemins partant du bourg ${ }^{18}$. Les petites boutiques se 
multiplient : même un marchand de CD s'est installé. L'arrivée de la première voiture a fait sensation. Son propriétaire tient à la laisser garée devant chez lui, au vu et au su de tous...

Incontestablement, le processus de formation de classes est très avancé. Beaucoup de ceux qui se situaient un peu au-dessus de la moyenne sont devenus "riches». La majorité a vu son sort s'améliorer. Mais même les autorités locales le reconnaissent : la pauvreté gagne du terrain, sans qu'il soit possible à ce stade d'estimer si, dans ce kebele, cette paupérisation est absolue ou relative. Pour la première fois, semble-t-il, une institution officielle a reconnu ce fait, qui pourtant sautait aux yeux depuis une dizaine d'années. "The very poorest have not seen improvements-to the contrary, even a worseningof consumption since 2005 ", admet - enfin - la Banque mondiale ${ }^{19}$. L'inflation a été particulièrement forte ces dernières années. La hausse des prix alimentaires a été de $22 \%$ en moyenne annuelle de 2005 à 2011 , et atteignait $39 \%$ cette dernière année ${ }^{20}$. Elle frappe particulièrement les paysans pauvres, qui produisent moins qu'ils ne consomment.

Ainsi un sentiment de classes, et leur antagonisme, commencent à émerger. D'un côté, on entend: "si je suis riche, ce n'est que grâce à mon travail; les pauvres n'ont qu'à s'en prendre à eux-mêmes". De l'autre: "les riches et les autorités se sont ligués pour nous déposséder ». La montée de ce sentiment dans les campagnes est totalement absente des analyses officielles, dont le parti au pouvoir est pourtant prolixe. Peut-être parce qu'elle révèlerait la face cachée du mode de développement rural que les autorités ont choisi, et peut être aussi parce que, depuis toujours, le régime considère que la société éthiopienne est d'abord structurée en nations/ethnies, les frontières de classes étant supposées moins prégnantes.

Par ailleurs, si l'agriculture a bien été le moteur d'un enrichissement catégoriel, celui-ci ne doit pratiquement rien à l'un des deux axes d'action du Developmental State: les cultures traditionnelles. De l'avis de tous les paysans et commerçants en grain, leur rendement stagne, la hausse de la consommation d'engrais ne faisant que compenser l'épuisement et l'érosion des sols. En revanche, les cultures de rente ont révolutionné le paysage agricole d'Armanya, notamment grâce au développement des infrastructures l'une des actions prioritaires du Developmental State-, à la croissance urbaine, en particulier d'une classe moyenne citadine, et aux efforts des autorités pour prendre pied dans le marché mondial.

Il faut insister aussi sur une révolution mentale. Depuis des siècles, le pouvoir reposait sur la possession de la terre. Dans un pays où l'activité quasi-unique était l'agriculture, l'étendue de la terre possédée déterminait le niveau de richesse, et donc l'accès au pouvoir. Réciproquement, le pouvoir donnait accès à la terre, attribuée par la couronne pour les services qui lui étaient rendus. Aujourd'hui, un paysan « riche» ne verra pas nécessairement d'un mauvais œil qu'aucun de ses enfants ne reprenne son métier trop dur, trop risqué, trop peu profitable et choisisse de devenir fonctionnaire ou " businessman ». Bien sûr, il leur transmettra ses droits fonciers, et ils mettront la terre en location ou métayage, pour arrondir leur fin de mois. Il a développé son exploitation comme un tremplin pour atteindre le seuil qui lui permet de s'enrichir ensuite dans les services. Le paysan " pauvre ", lui, est absolument convaincu que s'il a un avenir, c'est ailleurs. "Même si j'arrivais à m'en sortir, mes enfants ne le pourraient pas ", dit-il. " Donc autant que je supporte aujourd'hui les aléas et les sacrifices du départ plutôt que de les reporter demain sur toute ma famille». Mais pour aller où ? Deux à deux millions et demi de jeunes 
arrivent chaque année sur le marché du travail. Le principal gisement des futurs emplois, le secteur manufacturier, ne décolle pas. Il représente la même proportion du PIB que voilà un demi-siècle, à la fin du règne de Hailé Sélassié. Pour que le " miracle » éthiopien se poursuive, officiellement une croissance de $10 \%$ pendant les huit dernières années, en réalité autour de $6 \%$ à $7 \%$, l'économie devra se restructurer et le Developmental State re-cibler son mode et son périmètre d'intervention.

\section{BIBLIOGRAPHIE}

Aalen L., Tronvoll K., 2009. The end of democracy? Curtailing political and civil rights in Ethiopia. Review of African Political Economy, 36, 120, p. 193-207.

Bevan, P. 2010. The MDG-ing of Ethiopia's rural communities 2003-10: some meso, micro and macro consequences. Draft paper prepared for the Symposium on promoting social inclusion in South Asia: policies, pitfalls and analysis of welfare/insecurity regimes. Oxford, Mokoro.

Davis K. et al., 2010. In-depth assessment of the Public Agricultural Extension System of Ethiopia and recommendations for improvement. Discussion Paper 01041, Washington, DC: IFPRI.

Desalegn Rahmato., 2009. The Peasant and the State: studies in agrarian change in Ethiopia 1950s-2000s. Addis Ababa, Addis Ababa University Press.

Ege S., 1997. The promised land: the Amhara land redistribution of 1997. Working Papers on Ethiopian Development, No 12. Dragvoll, Norwegian University of Science and Technology.

EPRDF, 2002. The EPRDF's rural development vision: an overview. Addis Ababa, Special Issue No 3 of Renewal (Tehadso).

EPRDF, 2006a. Development, democracy and revolutionary democracy. Internal document, widely regarded as written by Meles Zenawi, Addis Ababa, EPRDF.

EPRDF, 2006b. EPRDF Programme. http://www.eprdf.org.et/EPRDFE/faces/document/ document.jsp. Addis Ababa, EPRDF.

Lefort R., 2005. A short survey of the relationship between powers - mengist - and peasants gebäre - in a peasant community of Northern Shoa. Nord-Süd Aktuell, 2, p. 211-221.

Lefort R., 2007. Powers - mengist - and peasants in rural Ethiopia : the May 2005 elections. Journal of Modern African Studies 45, 2, p. 253-276.

Lefort R., 2010. Power - mengist - and peasants in rural Ethiopia: the post 2005 interlude. Journal of Modern African Studies 48, 3, p. 435-460.

Meles Zenawi, 2006. African Development: dead ends and new beginnings. Addis Ababa, EPRDF internal document.

Ministry of Finance and Economic Development (MoFED), 2010. Growth and Transformation Plan 2010/11-2014/15 (GTP). Addis Ababa, MoFED. 
Planel, S., 2012. Impacts of agricultural extension policy on poverty and food insecurity: a critical approach. Abstract of a forthcoming communication at the $18^{\text {th }}$ International Conference of Ethiopian Studies, Dire Dawa, 29 October-2 November 2012.

Segers K. et al., 2009. Be like bees - the politics of mobilising farmers for development in Tigray, Ethiopia. African Affairs, 108/430, p. 91-109.

\section{NOTES}

1. L'Éthiopie est une république fédérale, composée de neuf régions, dont la région amhara, la deuxième par sa population, et de deux villes.

2. Le kebele est la plus petite unité administrative, correspondant grosso modo à ce qu'était la paroisse. L'unité supérieure est le district (woreda).

3. Le programme devrait toucher 10 millions d'Éthiopiens dans 411 woreda (sur un total d'un peu plus de 700), pour un budget de 3,6 milliards de dollars sur les cinq prochaines années. Ethiopian Radio and Television Agency, 02/10/2014.

4. Pour un débat sur le Developmental State, on peut se tourner vers De Waal A., 2013. The theory and practice of Meles Zenawi. African Affairs, 112/446, p. 148-155; Lefort R., 2014, puis A response to Alex de Waal. African Affairs et De Waal A. 2014, A response to René Lefort. African Affairs, 112/448, p. 460-470.

5. Armanya tient son nom d'un groupe de marchands arméniens qui s'y étaient installés, et dont aucune trace ne demeure.

6. Depuis 2003, l'auteur se rend en moyenne deux fois par an dans ce kebele pour y étudier principalement les rapports entre le pouvoir local et les paysans, y compris lors de périodes critiques, comme les élections de 2005. Voir en particulier Lefort R. 2007. Powers - mengist - and peasants in rural Ethiopia: the May 2005 elections. The Journal of Modern African Studies, Volume 45, Issue 02, p. 253-276.

7. En 1991, le Derg était militairement défait par une coalition de quatre mouvements à base ethnique, l'Ethiopian People's Revolutionary Democratic Front (EPRDF), dont le Tigray People's Liberation Front (TPLF) reste jusqu'à nos jours le pilier.

8. Ege S., 1999. The promised land: The Amhara land redistribution of 1997. Working Papers on Ethiopian Development, Trondheim, Centre for Environment and Development, University of Trondheim, $153 \mathrm{p}$.

9. Les statistiques éthiopiennes, à plus forte raison locales, sont au mieux fantaisistes, au pire, délirantes. Par exemple, en 2004, le kebele abritait officiellement un tout petit peu plus de 1000 ha cultivés, et, aux dires de tous, il ne restait pas la moindre parcelle exploitable. Quelques années plus tard, cette superficie devenait 1200 ha, sans aucune explication sur l'origine des 200 ha supplémentaires. En 2012 enfin, elle était portée à 1500 ha, "sur instruction des services officiels de statistiques", selon les autorités locales.

10. Meles Zenawi, Gimgema Paper, 2001, cité par Paulos Milkias, 2003. Ethiopia, the TPLF, and the Roots of the 2001 Political Tremor, Northeast African Studies Vol. 10, No. 2 (New Series), p. 13-66.

11. Tout ce processus est détaillé en particulier dans Lefort R. 2012. Ethiopia: Free Market Economy, 'Developmental State' and party-state hegemony: the case of the 'model farmers'. The Journal of Modern African Studies, Volume 50, Issue 04, p. 681-706.

12. Idem.

13. "Riche " est à mettre entre guillemets parce que cette richesse est toute relative. Lorsqu'on l'interroge à ce sujet, un paysan « riche » répond en général : « je suis riche parce que je peux donner à ma famille trois repas par jour, toute l'année, et même envoyer mes enfants au collège (highschool) ». 
14. En un an, de juillet 2012 à juillet 2013, 170000 Éthiopiennes ont légalement émigré vers la seule Arabie Saoudite, essentiellement comme bonne. Ethiopian Migrant Workers Return From Saudi With Tales of Abuse, Bloomberg, 13/12/2013.

15. Un paysan "riche" détaillait ainsi le budget d'un magasin de stockage qu'il venait d'achever : 100 dollars de matériaux divers, le bois de l'armature gratuit car coupé sur ses terres, son travail et le coup de main de quelques voisins. Il le loue 25 dollars par mois. L'investissement en capital est remboursé en quatre mois, le revenu annuel équivalent à celui d'une petite exploitation agricole...

16. di Nunzio M., 2014. Thugs, Spies and Vigilantes: Community Policing and Street Politics in Inner City Addis Ababa. Africa, 84/03, p. 444-465.

17. Ces observations rejoignent, mais pas toujours, les conclusions d'une recherche commencée en 1995 sur « change and continuity in rural Ethiopia », plus précisément sur « the long-term impact of development interventions in 20 rural Ethiopian communities». C'est le programme WIDE (Wellbeing and Illbeing Dynamics in Ethiopia). Pour en savoir plus, voir http://www.mokoro.co.uk/files/13/ file/CD_July2014.pdf

18. Sur les ravages de cet alcool, voir Yeraswork Admassie \& Ezana Amdework, 2010. The Araqe dilemma. The socioeconomics of traditional distillet alcohol production, marketing and consumption in Ethiopia. Forum for Social Studies, Addis Abeba.

19. Idem p. XVI.

20. World Bank Group 2015. Ethiopia - Poverty Assessment 2014. Report No. AUS6744, January 2015, p. 61.

\section{AUTEUR}

\section{RENÉ LEFORT}

René Lefort, rmlefort@gmail.com, est chercheur indépendant. Il a publié récemment :

- Un débat sur le "democratic developmental state" avec Alex de Waal, dans deux numéros successifs de African Affairs : De Waal A., 2013. The theory and practice of Meles Zenawi. African Affairs, 112/446, p. 148-155; Lefort R., 2014. A response to Alex de Waal - De Waal A. 2014. A response to René Lefort. African Affairs, 112/448, p. 460-470.

- Lefort R., 2014. Ethiopia, a leadership in disarray. OpenDemocracy, 4 juillet 2014, https:// www.opendemocracy.net/ren\%C3\%A9-lefort/ethiopia-leadership-in-disarray

- Lefort R., 2010. Power - mengist - and peasants in rural Ethiopia: the post 2005 interlude. Journal of Modern African Studies 48, 3, p. 435-460. 